\title{
DIGITAL TECHNOLOGY Improving the digital health of the workforce in the COVID-19 context: an opportunity to future-proof medical training
}

\author{
Authors: Yang Chen ${ }^{\mathrm{A}}$ and Amitava Banerjee ${ }^{\mathrm{B}}$
}

As well as its profound effects on healthcare and wider society, the COVID-19 pandemic will have far-reaching implications for the future training and professional development of healthcare workers and, in particular, doctors. While initial educational priorities focused on creating a more agile workforce with better cross-specialty skill-mix, attention must now shift to how our system can prepare a proportionate response that not only addresses the needs of the pandemic but also the underlying challenges of healthcare: multimorbidity, bridging the evidence-practice gap and delivering integrated, personalised medicine for all.

It is our contention that meeting such challenges will require a rapid upskilling of the digital capabilities of the healthcare workforce. In short, optimising the health of the nation will depend, in part, on improving the digital health of the workforce.

In this review, we examine how digital technology played its part in the COVID-19 response, and how fundamental changes to medical training are urgently needed in the context of a 'healthcare reset'. Familiarity with health informatics, data science and digital technology have to move to centre stage in order to future-proof our profession in the years to come. The people that deliver care are our systems' greatest asset, and at a time when change is accelerating, we cannot knowingly allow current and future colleagues to be ill-equipped to survive and thrive in the practice of medicine.

KEYWORDS: Data science, health informatics, digital, training

DOI: $10.7861 /$ fhj.2020-0162

\section{Introduction}

'Health informatics', 'data science' and 'digital technology' should be terms that all practicing clinicians are familiar with. However,

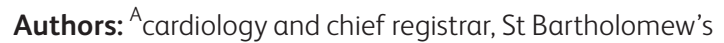
Hospital, London, UK; ${ }^{B}$ associate professor in clinical data science and honorary consultant cardiologist, St Bartholomew's Hospital, London, UK, University College London, London, UK and University College London Hospitals NHS Foundation Trust, London, UK this familiarity may be superficial or even coupled with feelings of apprehension and unease. As their definitions are overlapping and context-dependent, the concern is that descriptions such as 'the intelligent use of information and technology to provide better care for patients' may feel empty or devoid of meaning for many healthcare workers. ${ }^{1}$ There are parallels with how terms such as 'leadership' and 'management' were perceived in the past. Previous work highlighted how a set of unified competencies in informatics across medical specialties is lacking. ${ }^{2}$ Although, more recently, a universal health informatics competency framework has been launched by the Faculty of Clinical Informatics, there is clearly much more to do in order to increase the salience and impact of such work. ${ }^{3}$

As a result of the COVID-19 pandemic, there is now an opportunity to capitalise on the impetus to ensure that digital health technology continues to play a central and growing role in the future clinical model of healthcare delivery. An adequately trained workforce is a key tenet of any healthcare response, not only in times of crisis but also in longer-term planning and preparation for existing challenges. ${ }^{4}$ We propose that the vision of this response must be characterised by a digitally capable and digitally healthy workforce, one where all staff have universal digital literacy as a baseline, coupled with particular staffing groups who develop areas of domain expertise dependent on their different roles. We unpack the underlying rationale and suggested actions necessary to deliver such a vision, through evaluating three interlinking aims:

$>$ to highlight the central role that digital technology played during the COVID-19 response

$>$ to suggest educational strategies for clinicians in training on how to improve their digital health

$>$ to offer a perspective on future-proofing medical training, and the role of digital technology in healthcare delivery post-COVID.

\section{COVID-19: the digital health stimulus}

Digital technology played a key part in many different stages of the initial COVID-19 response (Fig 1). ${ }^{4}$ We wish to highlight three over-arching themes that this response demonstrates, all of which are vital considerations when trying to improve the future digital health of the workforce.

Firstly, the use of digital technology has cut across diverse organisations and necessitated collaborations between clinicians, academics, data scientists and policy makers. This must continue. 


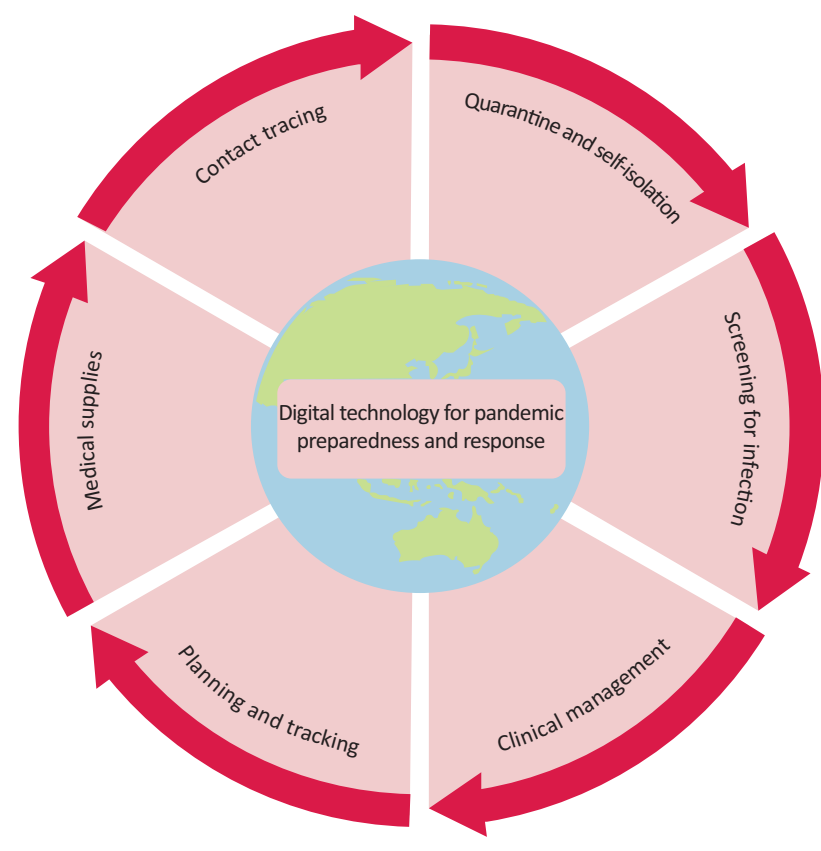

Fig 1. Digital technology as a tool for pandemic preparedness and response. Adapted with permission from Whitelaw S, Mamas MA, Topol E, Van Spall HGC. Applications of digital technology in COVID-19 pandemic planning and response. Lancet Digital Health 2020;2:e435-40.

These working relationships have been a core part of the response to COVID-19. On a system level, data linkage led by clinicians working with data scientists has generated insights with direct policy impact. ${ }^{5}$ An enduring proximity and efficient communication between multidisciplinary groups will be crucial in directing more nuanced policy responses associated with future waves of any pandemic. ${ }^{6}$

In addition, these relationships will be key to tackling existing healthcare challenges, which COVID-19 has exploited and accentuated. ${ }^{7}$ One recent exemplar case was of a multidisciplinary, artificial intelligence-enabled decision support tool deployed in an hyperacute stroke pathway demonstrated what can be achieved with digital technology. ${ }^{8}$

Secondly, although there are many other discrete success stories of digital technology in COVID-19, its use alone cannot be a panacea for all healthcare challenges. The recognition of its limits and its limitations are important. For example, during the pandemic, incomplete data has been a major obstacle; and furthermore, differing interpretations of existing or missing data held by various stakeholders has raised fundamental questions about how to better equip clinicians and leaders to be more objective and less biased when it comes to using data in prediction and forecasting.

From our own experiences, rapid healthcare expansion based on early modelling resulted in the deployment of the NHS Nightingale hospitals. ${ }^{9}$ However, their ongoing role and function was more limited and less adaptable than first envisaged. It is important to ask: 'Was it possible to harness data dashboards that could have allowed real-time visualisation of patient flows at a birds-eye view, thus facilitating a more unified response that allowed equitable attention to staffing, equipment and effort across all hospitals?'
The coupling of high-quality data and objective analysis are essential ingredients to enabling dynamic decision making that is trustworthy (whether clinically at the level of individuals or policywise at the level of populations). As the role of data visualisation and clinical decision support tools expands in medicine, the potential for frontline clinicians to be true 'custodians' of data in the 'learning health system' model will grow. ${ }^{10}$ The challenge therefore lies in ensuring that data science tools become a routine part of clinician training in order to drive their safe and effective implementation and that all clinicians recognise the limitations of models or decision support tools and the need to regularly review their data inputs and underlying assumptions. ${ }^{11}$

Thirdly, the pandemic saw digital technology play a role in the COVID-19 response that was distinct from the healthcare setting. The emotional reaction to the crisis on social media reflected the worldwide impact on health and society, with doctors and health professionals at the forefront. ${ }^{12}$ Social media (including Twitter and WhatsApp) were used among professionals to share initial experiences of local practice, as well as to disseminate new research findings. There were wider consequences of using such platforms to communicate public health messages, with opportunities and challenges alike for staff at all stages of their careers - from medical students through to members of the Scientific Advisory Group for Emergencies (SAGE) and shadow SAGE committees. One lesson of such messaging must be to reflect on our duties and responsibilities online when communicating our professional or personal opinions. Here, digital literacy and digital health of the workforce may extend to understanding the consequences of using such tools when expressing ourselves, recognising that the scalability of an online comment is far greater than a spoken one to a colleague at work.

The power of social media scalability was demonstrated in the Twitter analysis that spearheaded the retraction of two high-profile papers in The Lancet and NEJM based on concerns regarding the veracity and validity of source data. ${ }^{13}$ This final point perhaps best emphasises the urgent need to expand the number of engaged clinicians and researchers that will add to the 'wisdom of the expert crowd' that communicates with integrity.

Although many have already noted the strengths and weaknesses of Twitter compared with peer review in research, such a shift in influence from traditional sources of power (pay-wall academic journals) continues with growing speed, bringing opportunities and threats alike.

The future model of how clinical information and research findings are curated and shared will therefore represent the chance to progress toward greater transparency and accountability. Whether it is a study that examines an application of artificial intelligence (AI) or the possible flaws of using large routine datasets for observational analyses, all doctors will be required to have skills in data science and evidence-based medicine to objectively evaluate new and rapidly disseminated findings.

In terms of how such skills are taught, we have seen that during COVID-19, remote medical education from central sources as well as local initiatives has been a growing success story in terms of the democratisation of knowledge and the transfer of high quality, standardised teaching that is oriented around the learner rather than the teacher.

\section{Improving the digital health of the workforce}

For the reasons already mentioned, we see an inevitably growing demand for clinicians in training to develop a far greater understanding 
Box 1. Three tips for clinicians in training

\section{Practical evidence-based medicine skills}

These skills are directly transferrable to 'digital readiness'. First, we suggest 'actively reading papers' using frameworks such as the CONSORT reporting checklist for randomised controlled trials, which enables learning about the key steps in the conduct and reporting of trials, including data validity and methodology. ${ }^{14}$ If there are no journal clubs at your hospital, we suggest asking mentors and consultant colleagues to help set one up. In addition, do not underestimate the power of learning through doing. Taking part in a systematic review and/or a meta-analysis is an important way to put words into action.

> Escalation of IT concerns as opportunities to effect change A specific example is the accelerated remote access to electronic health record platforms during the pandemic, after long periods of inertia. This happened, in a major part, due to health professionals raising concerns about what was needed to provide virtual services during COVID-19. At our own trust, many individual acts of agency resulted in system change.

Beyond simple raising of concerns and being heard, leadership courses and adjunctive training initiatives such as the chief registrar scheme and the Topol digital fellowships are formal ways of promoting trainee curiosity, development and digital upskilling. ${ }^{15}$ These unique experiences receive consistently high feedback from trainees and we would encourage all trainees to explore these opportunities further, and to seek advice and support when applying to such schemes.

\section{$>$ Real data analysis}

Keep a logbook of the clinical decisions you make. Electronic health records have revolutionised the ability for clinicians to follow up their cases. Mastering how to apply Bayesian theory to decision making or how to interpret an investigation result that is out of keeping with the overall clinical picture requires experience allied with feedback from supervisors and, crucially, a personalised library of clinical cases, which can be used to self-reflect on how you were influenced or biased by patient, staff or external factors. It may additionally spark the idea of an audit or QI project that you wish to conduct which is driven by clinical need based on your experiences.

of the use of data, data analytics and its strengths and weaknesses within healthcare from the perspectives of research, education, clinical practice and healthcare policy.

This will require change on a system level but also change on a personal level. We provide some key, actionable tips for interested readers (Box 1), and discuss the urgency of formalising training across the workforce.

\section{Formalising digital training of the workforce}

The urgent prioritisation of training in data science can be argued independently of COVID-19. Fundamentally, the workflow of clinical practice had already reached an inflection point due to the pace of technological change. As outlined in The Topol Review and, more recently, the future doctor report in the realm of diagnostics and prognostication, AI will increasingly become part of the 'new normal', taking its place alongside early warning scores or electronic discharge summaries. ${ }^{16,17}$ The ability of the profession and the healthcare community to adequately react, evaluate and respond to such tools will require doctors in training to become as comfortable with AI algorithms as they currently are with physical examination.

Such a significant shift in the clinical model cannot be met with small incremental shifts in training or 'bolt on' optional courses and degrees. In order to ensure that the workforce survives and thrives in very different environments, data science and health informatics must be made to be central components of undergraduate and postgraduate education. Having more 'skin in the game' is not merely about risking other parts of medical education to ensure this new domain of knowledge is 'squeezed into curricula'; it is about the very identity of future doctors in training, and their place within the wider workforce.

\section{Blurred roles, blended skills and digital leaders}

In a system that is increasingly multidisciplinary, the role of the future doctor will not solely be determined by our own profession. How we integrate within an increasingly diverse workforce in order to best serve the patients that we look after as a team will require ongoing thought and evaluation.

We must build upon what still makes us unique, for example, our ability to make diagnoses or management plans without protocols or guidelines, our use of heuristics or first principles from detailed knowledge of pathophysiology and our handling of clinical risk and being the main executor of decisions. However, in future, we will see more members of the multidisciplinary team (supported by AI digital tools and decision-aids) take on roles that mimic the above. Thus, in a data-driven world, we need to evolve and adapt. Dedicated training that will confer greater in-depth knowledge and expertise of health informatics, data science, research methodology and statistics will represent key defining features of the future doctor. In the short term, by leveraging digital technology and its ability to crosscut every specialty, we foresee a set of national training programmes that would allow trainees to link together in ways that have not yet been realised. For example, novel collaborations and accelerated spread of best practice could occur through trainee leadership and followership. Locally, use of digital tools could allow trainees across specialties to set up communication platforms where audit and QI ideas are internally peer reviewed, strengthening the methodology of proposals and better aligning them with trust priorities, before seeking consultant support and/or formal registration and delivery. Tips could be shared regionally or even nationally, transforming such activities from a simple 'tick box' exercise to something far more educational for trainees and impactful to patients. ${ }^{17}$

\section{A call to arms}

We argue that even with these short-term innovations and other changes to medical training, this is not enough to 'move the dial' in a meaningful way. Thus, while schemes such as the Topol digital fellowship, NHS digital pioneers and NHS entrepreneurs represent positive worthy endeavours, they provide incremental shifts rather than paradigm change. The issue of self-selection additionally means that their benefits and changes may be unevenly distributed to only those who are ambitious enough to look in the first place, rather than a much more inclusive approach dedicated to entire cohorts. 
By way of emphasis, of the 336,059 doctors registered with the General Medical Council, 65,006 are in training and, every year, approximately 7,500 medical students enter our profession. ${ }^{18}$ Thus, even if a dedicated and embedded digital training scheme were offered to all undergraduates, it would still take considerable time for its effects to be felt across the profession.

Therefore, upgrading the digital health of the workforce must be all-encompassing and longitudinally embedded within continuous professional development from the start of undergraduate courses through to postgraduate training and lifelong learning. These changes will require constant focus in order to engage healthcare staff across specialties, hierarchies and professions.

Although calls to change medical education and training have been made before, there is finally a critical mass of independent factors to warrant and urge this to happen: an inflection point in technology, a global pandemic that has reset institutional and personal habits, and the overlay of a multimorbid population seeking a different relationship with healthcare. ${ }^{19}$

This last factor is the ultimate reason for such change, and as we seek to become more digitally enabled and digitally healthy ourselves, we must safeguard patients to ensure that we are doing things for the right reasons; innovation because it leads to demonstrable patient benefit rather than innovation because it is 'new and exciting'. The role of patient and public involvement in all aspects of the clinical model, including the training of healthcare staff, cannot be overstated and issues such as digital exclusion of patients will represent work that must dovetail with the other factors mentioned in this review.

\section{Conclusion}

Improving the digital health of all doctors and the wider workforce has overlap and synergy with other professional training and development initiatives. In summary, we emphasise the need to avoid conceptualising the digital health of the workforce in isolation. Rather, this endeavour should be seen as helping to improve a continuum of transferrable skills for future clinicians, useful in a spectrum of professional domains, be it academia, quality improvement, leadership or management.

The multifaceted role of future doctors could provide inherent 'value' to the healthcare system, measured in the currency of objective knowledge of health informatics, data science and digital technology, and fluency in the use, analysis and interpretation of such skills and tools. The imperative now is to work together and transform training to ensure that, as a profession, we realise this reality.

\section{References}

1 Health Careers. Health informatics. NHS. www.healthcareers.nhs. uk/explore-roles/health-informatics [Accessed 20 July 2020].

2 Jidkov L, Alexander M, Bark $P$ et al. Health informatics competencies in postgraduate medical education and training in the UK: a mixed methods study. BMJ Open 2019;9:e025460.
3 Faculty of Clinical Informatics. Competency Framework for Clinical Informaticians. FCI. https://facultyofclinicalinformatics.org.uk/corecompetency-framework [Accessed 04 August 2020].

4 Whitelaw S, Mamas MA, Topol E, Van Spall HGC. Applications of digital technology in COVID-19 pandemic planning and response. Lancet Digital Health 2020;2:e435-40.

5 Banerjee A, Pasea L, Harris S et al. Estimating excess 1-year mortality from COVID-19 according to underlying conditions and age in England: a rapid analysis using NHS health records in 3.8 million adults. Lancet 2020;395:1715-25.

6 The Academy of Medical Sciences. Prepare now for a winter COVID-19 peak, warns Academy of Medical Sciences. The Academy of Medical Sciences, 2020. https://acmedsci.ac.uk/more/ news/prepare-now-for-a-winter-covid-19-peak-warns-academy-ofmedical-sciences [Accessed 15 ]uly 2020].

7 Horton R. Offline: Don't let COVID-19 divert us completely. Lancet 2020;395:1534.

8 Nagaratnam K, Harston G, Flossmann E et al. Innovative use of artificial intelligence and digital communication in acute stroke pathway in response to COVID-19. FHJ 2020;7:169-73.

9 Bohmer R, Shand J, Allwood D et al. Learning systems: Managing uncertainty in the new normal of COVID-19. NEJM Catal Innov Care Deliv 2020:10.1056/CAT.20.0318.

10 33N. About 33N. 33N. www.33n.co.uk/about

11 Clinically Led workforce and Activity Redesign Programme. A novel initiative empowering clinical staff to use NHS data to evidence how we care for patients and redefine the workforce. CLEAR. https://clear-uk.org.uk/home

12 Kumar A, Khan SU, Kalra A. COVID-19 pandemic: a sentiment analysis. Eur Heart J 2020:ehaa597.

13 Joseph A. Lancet, New England Journal retract Covid-19 studies, including one that raised safety concerns about malaria drugs. STAT, 2020. www.statnews.com/2020/06/04/lancet-retracts-majorcovid-19-paper-that-raised-safety-concerns-about-malaria-drugs

14 Schulz KF, Altman DG, Moher D, CONSORT Group. CONSORT 2010 statement: updated guidelines for reporting parallel group randomised trials. BMJ 2010:340:c332.

15 Health Education England. Digital Fellowships. NHS, 2020. https:// topol.hee.nhs.uk/digital-fellowships [Accessed 05 August 2020].

16 Health Education England. The Topol Review. NHS, 2019. https:// topol.hee.nhs.uk/the-topol-review [Accessed 24 July 20].

17 Health Education England. Future doctor: A co-created vision for the future clinical team. NHS, 2020. www.hee.nhs.uk/our-work/ future-doctor [Accessed 02 August 2020].

18 Woolf K, Page M, Viney R. Assessing professional competence: a critical review of the Annual Review of Competence Progression. J $R$ Soc Med 2019;112:236-44.

19 General Medical Council. Key stats from the medical register. GMC 2020. https://data.gmc-uk.org/gmcdata/home/\#/reports/The \% 20 Register/Stats/report [Accessed 20 July 2020].

20 Prasad V. Perspective: beyond storytelling in medicine: an encounter-based curriculum. Acad Med. 2010;85:794-8.

Address for correspondence: Dr Amitava Banerjee, Institute of Health Informatics, University College London, 222 Euston Road, London NW1 2DA, UK. Email: ami.banerjee@ucl.ac.uk 